\title{
Hemolytic Uremic Syndrome in a Child with Burn Injuries
}

\author{
Sherif Emil, MD, CM, ${ }^{a}$ Richard Rockstad, PAC, ${ }^{b}$ \\ and David Vannix, MD, FACS, ${ }^{\mathrm{a}, \mathrm{b}}$ \\ Loma Linda and San Bernardino, California
}

\begin{abstract}
We report the first case of hemolytic uremic syndrome in a patient with burns. An unusual case of the syndrome developed in a 1-year-old black girl hospitalized after second-degree burns to $33 \%$ of her total body surface area. Acute abdominal distention, hemolytic anemia, hematuria, and oliguric renal failure developed 1 week after admission to the burn unit. Blood cultures grew Escherichin coli 0157:H7. She received supportive care and antibiotics, in addition to low-dose dopamine, which promptly reversed the oliguria. Dialysis was not required, and the child made a complete recovery. (J Burn Care Rehabil 1998;19:135-7)
\end{abstract}

Hemolytic uremic syndrome (HUS) is the most common cause of acute renal failure in childhood. ${ }^{1}$ This heterogenous disease typically follows an acute diarrheal illness and presents with a triad of microangiopathic hemolytic anemia, thrombocytopenia, and renal failure. The surgeon is often called on to evaluate an acute condition in the abdomen in these patients and to provide a route for peritoneal or hemodialysis. $^{2}$ Intestinal gangrene, perforation, intussusception, or stricture may necessitate operation. ${ }^{2,3}$ To our knowledge, HUS has not been reported in association with trauma or burns. In addition to this association, our patient displayed several other unique characteristics.

\section{CASE REPORT}

A I-year-old, previously healthy black girl was transferred from an outside facility to the regional burn unit after sustaining second-degree burns from scalding hot water to her face, back, hands, abdomen, and knees, totaling $33 \%$ of her body surface area. On admission, the child weighed $9.7 \mathrm{~kg}$, was afebrile, and had stable vital signs. She was started on prophylactic penicillin G, multiple vitamins, ranitidine, and rou-

From the "Department of Surgery, Loma Linda University School of Medicine, Loma Linda, and the "Inland Conntics Regional Bum Center, San Bernardino.

Reprint requests: Davia Vannix, $M D$, Department of Surgery, San Bernardino County Medical Center; 780 E. Gillert St., San Bernardino, $C A 92415$

Copyright (9) 1998 by the American Burn Association.

$0273-8481 / 98 / \$ 5.00+0 \quad 30 / 1 / 80821$ tine burn wound care with silver sulfadiazine dressings. Within 48 hours, the child had good bowel sounds, and nasogastric tube feedings with Pediasure (Abbott Laboratories, Columbus, Ohio) were started.

On the eighth hospital day, the child spiked a fever of $41.3^{\circ} \mathrm{C}$ with a heart rate of 160 to 190 beats per minute. Watery, nonbloody diarrhea, and abdominal distention developed, without tenderness or guarding, and grunting respirations. She was not tolerating feedings. Arterial blood gases showed normal oxygenation and ventilation. A complete blood count revealed a white blood cell (WBC) count of $11,000 /$ $\mathrm{mm}^{3}$ without a left shift, and a hemoglobin of 8.7 $\mathrm{gm} / \mathrm{dl}$. An abdominal series showed a normal chest radiograph and decreased gas in the intestine without gas-fluid levels, dilated loops, or free air. Urine, stool, sputum, and blood cultures were sent. The child's wounds were all clean and healing well. Nasogastric feeding was stopped, and intravenous hydration was started. A pediatrician evaluated the child and ordered gentamicin and ticarcillin for presumed sepsis of unknown origin.

On the eleventh hospital day, the patient appeared extremely ill. Fever and tachycardia persisted, and respiration increased to a rate of 28 to 48 breaths per minute, with grunting and intercostal retractions. An arterial blood gas on room air showed respiratory alkalosis and $\mathrm{PaO}_{2}$ of 78 torr. The diarrhea persisted. The abdomen was distended, tense, and with decreased bowel sounds, but nontender. An acute abdominal series showed signs of fluid overload in the 
lungs and a nongaseous abdomen with a few dilated loops. A diatrizoate sodium enema had normal results. Oliguria developed, with an average urine output of $0.25 \mathrm{ml} / \mathrm{kg} / \mathrm{h}$. The child had eyelid and extremity edema and an albumin level of $1.9 \mathrm{gm} / \mathrm{dl}$. Gross hematuria also developed, and the urinalysis showed +++ protein, 5 to $20 \mathrm{WBC}$ high power field (HPF), too-numerous-to-count red blood cells (RBCs), a few granular casts, and no bacteria. The WBC count climbed to $19,500 / \mathrm{mm}^{3}$ with a left shift, and the hemoglobin dropped to $4.3 \mathrm{gm} / \mathrm{dl}$. Platelet count was normal. The blood smear showed evidence of microangiopathic hemolytic anemia with numerous target cells and schistocytes. Electrolytes were normal. Stool, urine, and sputum cultures were negative, but blood cultures grew Escherichia coli. Based on this clinical syndrome, a diagnosis of HUS was made.

Supportive care continued, with urinary output monitoring, nasogastric decompression, carcful fluid and electrolyte management, packed RBC transfusion, and total parenteral nutrition. Ticarcillin was discontinued and ceftazidime was added. Furosemide boluses $(0.5$ to $1 \mathrm{mg} / \mathrm{kg}$ intravenously) were used twice for diuresis, but oliguria would recur within 2 to 3 hours of each dose. A dopamine drip at 3 $\mu \mathrm{g} / \mathrm{kg} / \mathrm{h}$ was started 48 hours after oliguria was first detected. Within 2 hours of starting the infusion, the urine output climbed to $1 \mathrm{ml} / \mathrm{kg} / \mathrm{h}$. Twenty-four hours after the infusion, the urine output was steady and averaging $3 \mathrm{ml} / \mathrm{kg} / \mathrm{h}$. Dopamine was discontinued without recurrence of the oliguria. A continuous upward trend in the blood urea nitrogen and creatinine levels during the oliguric phase was also arrested and reversed after the dopamine infusion.

The child went on to make a remarkable recovery. By the fourteenth hospital day, she had defervescence and her tachycardia had resolved. Respiratory function spontaneously improved as the abdominal distention and volume overload resolved. All renal and hematologic abnormalities also resolved. Her wounds made excellent progress without any need for operative interventions. She was discharged 23 days after hospitalization in good condition, tolerating a regular diet for age. Serotyping of the cultured E. coli confirmed the organism to be of the 0157:H7 type.

\section{DISCUSSION}

The pathophysiology of HUS has been thoroughly studied since the disease was first reported in 1955. ${ }^{1-6}$ HUS is an acute illness, most commonly occurring as a sequel to an enteric infection with $E$. coli 0157 :
H7. ${ }^{4,5}$ This organism, like several others, elaborates a cytotoxin or verotoxin, which gains access to the systemic circulation through the injured intestinal mucosa. The fundamental lesion of the disease is a thrombotic microangiopathy, which consists of endothelial injury and microthrombi. ${ }^{1-3}$ The renal glomeruli bear the brunt of the injury, resulting in hematuria and oliguria. Intravascular hemolysis and thrombocytopenia are the two next most common consequences. Whereas RBC transfusions are frequently necessary, thrombocytopenia is rarely severe enough to require platelet transfusions and, uncommonly, may be absent altogether. ${ }^{5}$ Gastrointestinal complications include pancreatitis, rectal prolapse, intestinal stricture, perforation, or intussusception, segmental colonic gangrene, and hepatic damage. ${ }^{3}$ However, most patients do not require operative intervention if the correct diagnosis is made. In fact, Tapper et al. ${ }^{2}$ suggest that all patients with bloody diarrhea should undergo microbiologic evaluation for $E$. coli $0157: \mathrm{H} 7$ before any surgical intervention, to avoid unnecessary operation. In retrospect, the abdominal findings in our patient were secondary to the enteric infection and the ascites resulting from oliguria and hypoalbuminemia. An atypical, less common form of HUS, nondiarrheal or $D$-, can also occur and is associated with a poor prognosis.?

This case provides several important lessons in the diagnosis and management of HUS. The average incubation period of $E$. coli $0157: \mathrm{H} 7$ is 4 days and can be as long as 2 weeks. ${ }^{3,4}$ HUS is known to be endemic in California, among other areas. ${ }^{3,8} \mathrm{It}$ is likely that this patient was an asymptomatic carrier of the organism until she suffered the critical injury. The intestinal ileus and generalized immunosuppression associated with severe burns may have allowed the organism to translocate across the gastrointestinal mucosa and result in a systemic infection.

The organism was recovered from the blood, not the stool. It is not unusual for the organism to evade isolation from the stool, especially because the period between the onset of diarrhea and the obtaining of blood cultures is prolonged. ${ }^{3,4}$ However, it is unclear why the literature does not recommend blood cultures. Perhaps positive blood cultures are rare, or the lack of serious systemic signs does not prompt a search for the organism in the blood. In this patient, with a prominent systemic illness, culturing the organism from the blood helped confirm the diagnosis of HUS.

By the same token, the use of two intravenous antibiotics (gentamicin and ceftazidime), to which the organism was sensitive, appeared to be very effec- 
tive in clearing the infection and aiding the patient's recovery. This is an important observation, in light of the controversy surrounding the use of antibiotics in HUS. The literature does not recommend routine antibiotic use and some believe that bactericidal antibiotics may increase verotoxin elaboration and worsen the syndrome. ${ }^{8}$ Positive blood cultures and prominent systemic signs may therefore be exceptions to the general recommendation of avoiding antibiotics.

The platelet count does not correlate with prognosis in HUS and is not a risk factor for the severe form of the syndrome. ${ }^{8}$ Thrombocytopenia never developed in our patient. Whether the absence of thrombocytopenia predicts a good prognosis, as in this case, remains to be determined as further clinical experience with HUS accumulates.

Our patient received furosemide and low-dose dopamine for oliguria. Furosemide was effective only transiently, but dopamine appeared to result in immediate and lasting improvement in renal function. Recent reviews recommend early dialysis for oliguria associated with HUS. ${ }^{1,3}$ To our knowledge, dopamine has not been reported in the treatment of HUS. There is a good pathophysiologic explanation for the success of dopamine. Renal arterial and arteriolar involvement and narrowing may predominate in at least some cases of HUS. ${ }^{1,7}$ In any case, it may be prudent to use a brief trial of dopamine before proceeding to dialysis.

In conclusion, this case alerts us to the possible occurrence of HUS in critically injured patients, and provides us with valuable lessons in the diagnosis and management of this disorder.

\section{REFERENCES}

1. Frishberg Y, Obrig TG, Kaplan BS. Hemolytic uremic syndromc. In: Holliday MA, Barraft TM, Avner ED, editors. Pediatric nephrology. Baltimore, Williams and Wilkins, 1994: 871-87.

2. Tapper D, Tarr P, Avner E, Brandt J. Waldhausen. Lessons learned in the management of hemolytic uremic syndrome in children. J Pediatr Surg 1995;30:158-63.

3. Robson WLM, Leung AKC, Kaplan BS. Hemolytic-uremic syndrome. Curr Probl Pediatr 1993;23:16-33.

4. Cleary TG. Cytotoxin-producing Escherichia coli and the hemolytic uremic syndrome. Pediatr Clin North Am 1988;35: 485-501.

5. Levin M, Walters MDS, Barran TM. Hemolytic uremic syndrome. Adv Pediatr Infect Dis 1989;4:51-81.

6. Siegler RL. Management of hemolytic-uremic syndrome. J Pediatr 1988;112:1014-20.

7. Fitzpatrick MM, Waiters MDS, Trompeter RS, Dillon MJ, Barratt TM. Atypical (non-diarrhea-associated) hemolytic-uremic syndrome in childhood. J Pediatr 1993;122:532-7.

8. Rowe PC, Orrbine E, Wells GA, McLaine PN. Epidemiology of hemolytic-uremic syndrome in Canadian children from 1986 to 1988. J Pediatr 1991;119:218-24. 